\title{
Haemophilus influenzae biofilms in primary ciliary dyskinesia: a moving story
}

\author{
Amelia Shoemark ${ }^{1,2}$ \\ Hospital, London, UK. \\ Correspondence: Amelia Shoemark. E-mail: a.shoemark@adundee.ac.uk
}

Affiliations: 'School of Medicine, University of Dundee, Dundee, UK. ${ }^{2}$ Dept of Paediatrics, Royal Brompton

@ERSpublications

Impaired motile cilia function in the PCD cells results in susceptibility to NTHi biofilm development http://ow.ly/9D2u30eamR6

Cite this article as: Shoemark A. Haemophilus influenzae biofilms in primary ciliary dyskinesia: a moving story. Eur Respir J 2017; 50: 1701369 [https://doi.org/10.1183/13993003.01369-2017].

\begin{abstract}
Mucociliary clearance is a first line of defence against airway infection. Motile cilia function is important for the transport of inhaled bacteria and other unwanted particulate matter away from the lungs. Primary ciliary dyskinesia (PCD) is an inherited condition in which motile cilia function is disrupted. Patients with PCD suffer from poor mucociliary clearance of the upper and lower airways leading to chronic wet cough, rhinusinusitis, otitis media and reoccurring infections [1]. The most commonly isolated organism from PCD sputum or nasal lavage is Haemophilus influenzae [1]. Non-typeable Haemophilus influenzae (NTHi) have been shown to form biofilms in the adenoids and middle ear of children with otitis media [2, 3]. The study by WALKER et al. [4] in this issue of the European Respiratory Journal poses the question: is the respiratory epithelium in PCD susceptible to NTHi biofilms due to motile ciliary dysfunction?
\end{abstract}

WALKER et al. [4] investigate NTHi biofilm formation in vitro on air liquid interface cultures of the nasal epithelium from healthy controls and patients with PCD [5]. The authors conclude that impaired motile cilia function in the PCD cells results in susceptibility to NTHi biofilm development. After $72 \mathrm{~h}$ in an epithelial cell co-culture, nearly twice the number of viable NTHi are found on PCD epithelial cultures compared to healthy control cultures. Furthermore imaging with confocal and scanning electron microscopy show that NTHi are more likely to form aggregates, believed to be biofilms, on the PCD cells [4].

Poor ciliary function resulting in enhanced biofilm formation in this model is not unexpected given previous in vivo evidence that poor ciliary function is related to infection $[6,7]$. Beyond respiratory medicine, in the field of material science, the idea that ciliary motion can reduce biofilm formation has been exploited for some time and bioengineered materials which emulate cilia beating to help prevent biofilm build up have been created for use in the shipping industry $[5,8]$. Unlike ships, people have an innate immune system which will inevitably play a role in bacterial biofilm formation. The authors of this study acknowledge that the role of the circulating immune system is not considered within this co-culture system and, therefore, the relative contribution of motile ciliary dysfunction to NTHi susceptibility cannot be completely ascertained. The authors do, however, consider local airway defences beyond motile cilia and show that PCD and non-PCD epithelial cells produce similar levels of cytokines and have similar nitric oxide expression [4]. The suggestion is, in this model, that the motile cilia driven mucociliary impairment is the most likely explanation for the increased biofilm formation on the surface of the PCD cells.

These findings raise interesting questions regarding the relationship between nitric oxide, motile cilia and infection. Nitric oxide is a ubiquitous, bioactive, highly diffusible gas with multiple functions within the airway, including bronchodilation and host defence. In 1994 it was found that individuals with PCD have

Received: July 072017 | Accepted: July 102017

Conflict of interest: None declared.

Copyright OERS 2017 
approximately 10-fold lower levels of nitric oxide gas in the paranasal sinuses than their non-PCD counterparts [9]. This finding was rapidly exploited for PCD diagnostics and measurement of nasal nitric oxide is now a first line recommended test contributing to a diagnosis of PCD [10]. Nasal nitric oxide is not diagnostic alone and can be low for other reasons, such as smoking or the presence of nasal polyps; it is also unsuitable for testing in babies and, occasionally, individuals with PCD have normal nitric oxide levels. Both these scenarios are demonstrated in subjects recruited for the study by WALKer et al. [4] (e.g. subject PCD 005 and 013 respectively)

The reason for low nitric oxide is one of the curiosities of PCD diagnostics and no hypotheses put forward have yet been conclusively confirmed [11]. All three isoforms of the nitric oxide synthase enzyme thought to generate nitric oxide in the airways (eNOS, iNOS and nNOS) are present in the ciliated epithelium and low nitric oxide cannot be entirely explained by inflammation, mucus or anatomical differences in the sinuses [10]. Previous studies have suggested that cultured epithelial cells taken from individuals with PCD produce a lower level of nitric oxide in response to infection [12], and that following infection PCD epithelial cells express reduced levels of inducible nitric oxide compared to controls [13], but the literature remains conflicting [14]. The present study adds to this debate by showing no difference in nitric oxide production in PCD and non-PCD epithelial cells following NTHi infection in a co-culture model [4].

Interestingly, having presented the problem that epithelial cells with compromised motile cilia function are more likely to develop NTHi biofilms, WALKER et al. [4] suggest a potential solution by considering the bactericidal role of nitric oxide in the airways. The authors show that the use of exogenous nitric oxide therapy combined with azithromycin reduces bacterial load significantly more than the use of antibiotic alone. WALKER et al. [4] suggest increasing nitric oxide in PCD airways could facilitate bacterial dispersal and killing by antibiotics. Based on the heterogeneity of PCD, the small number of patients studied, the limited number of strains of NTHi, and considering the possible adverse effects of breaking up a biofilm, this is far from guaranteed to be effective in the clinic, but further investigation of this suggestion could be warranted. Until recently, sufficiently powered clinical trials in PCD were virtually impossible. However, like the biofilms themselves, over the last few years, PCD researchers and clinicians have become organised as a community, working together as part of large European projects such as BEST CILIA (http://bestcilia. $\mathrm{eu} /$ ) and BEAT PCD (www.beatpcd.org/). This has resulted in large patient cohorts, such as the PCD registry and the iPCD cohort [15] The first evidence based diagnostic guidelines were published in the European Respiratory Journal earlier this year [10], and the first multicentre clinical trials for therapies have been conducted $[16,17]$. These well-defined cohorts and networks will provide insight into disease progression and present the opportunity to gather evidence for PCD specific treatments. Hopefully, by using large patient cohorts, some of the questions raised by this study will be investigated in vivo: for example, are patients with PCD susceptible to NTHi biofilms? Why is nitric oxide reduced in PCD? And will nitric oxide supplementation ultimately prove to be a useful therapy?

\section{References}

1 Shah A, Shoemark A, MacNeill SJ, et al. A longitudinal study characterising a large adult primary ciliary dyskinesia population. Eur Respir J 2016; 48: 441-450.

2 Hall-Stoodley L, Hu FZ, Gieseke A, et al. Direct detection of bacterial biofilms on the middle-ear mucosa of children with chronic otitis media. JAMA 2006; 296: 202-211.

3 Nistico L, Kreft R, Gieseke A, et al. Adenoid reservoir for pathogenic biofilm bacteria. J Clin Microbiol 2011; 49: $1411-1420$.

4 Walker WT, Jackson CL, Allan RN, et al. Primary ciliary dyskinesia ciliated airway cells show increased susceptibility to Haemophilus influenzae biofilm formation. Eur Respir J 2017; 50: 1700612.

5 Liu F, Ramachandran D, Urban MW. Colloidal films that mimic cilia. Adv Funct Mater 2010; 20: 3163-3167.

6 McKenzie CW, Klonoski JM, Maier T, et al. Enhanced response to pulmonary Streptococcus pneumoniae infection is associated with primary ciliary dyskinesia in mice lacking Pcdp1 and Spef2. Cilia 2013; 2: 18.

7 Pittet LA, Hall-Stoodley L, Rutkowski MR, et al. Influenza virus infection decreases tracheal mucociliary velocity and clearance of Streptococcus pneumoniae. Am J Respir Cell Mol Biol 2010; 42: 450-460.

8 Shivapooja P, Wang QM, Orihuela B, et al. Bioinspired surfaces with dynamic topography for active control of biofouling. Adv Mater Weinheim 2013; 25: 1430-1434.

9 Lundberg JON, Weitzberg E, Nordvall SL, et al. Primarily nasal origin of exhaled nitric oxide and absence in Kartagener's syndrome. Eur Respir J 1994; 7: 1501-1504.

10 Lucas JS, Barbato A, Collins SA, et al. European Respiratory Society guidelines for the diagnosis of primary ciliary dyskinesia. Eur Respir J 2017; 49: 1601090.

11 Walker WT, Jackson CL, Lackie PM, et al. Nitric oxide in primary ciliary dyskinesia. Eur Respir J 2012; 40: 1024-1032.

12 Smith CM, Fadaee-Shohada MJ, Sawhney R, et al. Ciliated cultures from patients with primary ciliary dyskinesia do not produce nitric oxide or inducible nitric oxide synthase during early infection. Chest 2013; 144: 1671-1676.

13 Pifferi M, Bush A, Maggi F, et al. Nasal nitric oxide and nitric oxide synthase expression in primary ciliary dyskinesia. Eur Respir J 2011; 37: 572-577.

14 Walker WT, Jackson CL, Coles J, et al. Ciliated cultures from patients with primary ciliary dyskinesia produce nitric oxide in response to Haemophilus influenzae infection and proinflammatory cytokines. Chest 2014; 145: 668-669. 
15 Goutaki M, Maurer E, Halbeisen FS, et al. The international primary ciliary dyskinesia cohort (iPCD Cohort): methods and first results. Eur Respir J 2017; 49: 1601181

16 Paff T, Daniels JM, Weersink EJ, et al. A randomised controlled trial on the effect of inhaled hypertonic saline on quality of life in primary ciliary dyskinesia. Eur Respir J 2017; 49: 1601770.

17 Kobbernagel HE, Buchvald FF, Haarman EG, et al. Study protocol, rationale and recruitment in a European multi-centre randomized controlled trial to determine the efficacy and safety of azithromycin maintenance therapy for 6 months in primary ciliary dyskinesia. BMC Pulm Med 2016; 16: 104. 